\title{
Autoconcepto del Estudiante en Psicología: Una Perspectiva Multidimensional
}

\author{
Student Self-Concept in Psychology: A \\ Multidimensional Perspective
}

Gerardo Peña-Loaiza Universidad Técnica de Machala gpena@utmachala.edu.ec https://orcid.org/0000-0002-2150-2988

Yadira Sánchez-Padilla Universidad Técnica de Machala ysanchez@utmachala.edu.ec https://orcid.org/0000-0002-8380-6434

Carlos Carpio-Mosquera Universidad Técnica de Machala ccarpio@utmachala.edu.ec https://orcid.org/0000-0001-6335-1922

Rosa Salamea-Nieto Universidad Técnica de Machala rsalamea@utmachala.edu.ec https://orcid.org/0000-0001-5315-3439

Revista Cumbres Vol.7 №2

Versión electrónica ISSN 1390-3365

http://investigacion.utmachala.edu.ec/revistas/index.php/Cumbres http://doi.org/10.48190/cumbres.v7n2a3 


\title{
RESUMEN
}

El presente trabajo tuvo como finalidad evaluar el autoconcepto del estudiante en psicología desde una perspectiva multidimensional, mediante la aplicación del test AF-5 (Autoconcepto Forma 5) a una muestra de 450 estudiantes de las carreras de Psicología Clínica y Psicopedagogía de la Universidad Técnica de Machala. Se utilizó una metodología descriptiva de diferencia de grupos, de corte transversal con enfoque cuantitativo. Los participantes presentaron niveles altos de autoconcepto en las dimensiones académico-laboral y físico, no así en las dimensiones social y emocional. Se encontraron algunas diferencias entre los estudiantes de los semestres inferiores y superiores, en lo concerniente a la dimensión emocional y únicamente en la dimensión familiar se revelaron diferencias significativas relacionadas al sexo y a la carrera estudiada. En relación a la edad no hubo diferencias significativas en ninguna de las dimensiones evaluadas.

Palabras clave: Autoconcepto multidimensional, estudiante universitario, psicología.

\begin{abstract}
The purpose of the present work was to evaluate the student's self-concept in psychology from a multidimensional perspective, by applying the AF-5 test (Self-concept Form 5) to a sample of 450 students from the careers of clinical psychology and psychopedagogy of the Technical University of Machala. A descriptive methodology of group difference was used, cross-sectional with a quantitative approach. The participants increased high levels of self-concept in the academic-work and physical dimensions, but not in the social and emotional dimensions. Some differences were found between the students of the lower and upper semesters, regarding the emotional dimension and only in the family dimension, significant differences related to sex and the career studied were revealed. In relation to age there were no significant differences in any of the dimensions evaluated.
\end{abstract}

Keywords: multidimensional self-concept, university student, psychology. 


\section{INTRODUCCIÓN}

A lo largo de la historia, desde diferentes áreas como la psicología, la pedagogía, la sociología, filosofía y la educación en general, se ha intentado explicar cómo las personas se conciben así mismas; de ello ha devenido un amplio cuerpo de investigaciones acerca del autoconcepto y su importancia como una variable en la identidad de las personas (López et al., 2016). Como resultado de estas investigaciones se ha podido definir el autoconcepto como un conjunto de representaciones mentales, construidas por el individuo a partir de sus experiencias personales tanto positivas como negativas y las valoraciones del entorno, que evidencia una percepción clara del yo en diferentes facetas y momentos de vida, autorregulando su comportamiento (Marsh y Shavelson, 1985; Mato, et al., 2020).

Shavelson et al., (1976) desarrollaron un modelo de autoconcepto multidimensional categorizándolo jerárquicamente en dos dominios: autoconcepto académico y autoconcepto no académico. El autoconcepto académico hace referencia a las percepciones que tiene el individuo sobre sus capacidades en las diferentes materias escolares; mientras que el autoconcepto no académico se divide a su vez en los subdominios social, físico y personal (López, et al., 2016). El dominio social hace referencia a las percepciones que tiene el individuo sobre sus habilidades sociales y del grado de aceptación social que posee; el dominio físico se refiere a las percepciones sobre la apariencia física y habilidades físicas, y el dominio personal a las percepciones que tiene el individuo en cuanto a su identidad como un ser individual y de sus estados emocionales.

Palacios-Garay y Coveñas-Lalupú (2019), reconocen además las dimensiones familiar y emocional del autoconcepto; refiriéndose a la primera de ellas como la percepción que tiene la persona de su participación en el entorno familiar, así como de su aceptación y pertenencia, lo que puede experimentarse en un sentido tanto positivo como negativo; y la segunda dimensión, como la percepción que tiene el individuo sobre su situación emocional y su respuesta al entorno.

La complejidad del autoconcepto se revela en el número y la diversidad de categorías que el individuo construye con base en las autopercepciones obtenidas a partir de sus experiencias vividas en su interacción con otras personas; en este sentido, el autoconcepto de alguien, consistiría en la totalidad de las representaciones cognitivas individuales que tiene esa persona sobre sí mismo y los diferentes autoconceptos de esa persona serían los subconjuntos de tales representaciones que se relacionan con diferentes atributos del yo (Pekrun y Stephens, 2015). Según Obregón-Brocher et al., (2020), el autoconcepto afecta tanto las percepciones de ciertas características individuales, así como la formación de la identidad global.

Penado y Rodicio (2017), resaltan que el autoconcepto es dinámico y corresponde al sistema de autopercepciones que tiene un individuo sobre cada 
uno de los dominios; de esta manera, los distintos dominios o dimensiones que forman el autoconcepto no se sitúan en un mismo plano de importancia ni mantienen siempre el mismo nivel de jerarquía, sino que se ordenan de acuerdo a su relevancia con base en un sistema subjetivo de valoraciones que lleva a cabo el individuo en un momento dado y que está determinado por algunas variables como: la edad de la persona, su personalidad, intereses, actitudes, valores, etc., además de la influencia que puedan ejercer la cultura y los otros significativos (Cazalla y Molero, 2013; Cole et al., 2001; González, 2005).

La importancia del estudio del autoconcepto radica en el hecho de que este constructo explica otras variables psicológicas y de la personalidad, sustanciales para el ajuste a la vida y el bienestar emocional del individuo (Chaparro-Aguado, 2020); de esta manera, el autoconcepto podría promover motivación y ajuste comportamental necesario para alcanzar el éxito en diferentes contextos y áreas del desenvolvimiento humano (Esnaola, et al., 2008).

Por otra parte, la relación del autoconcepto con el autoestima (Garaigordobil y Durá, 2005), la autoeficacia (Jansen, et al., 2015), el locus de control (Sagone y De Caroli, 2014), la inteligencia emocional (Campbell, et al, 1996) y los factores de personalidad (Pilarska, 2018) han sido el centro de muchas investigaciones en las últimas décadas; asimismo, los estudios realizados en sujetos que viven situaciones de transición ya sea por factores biológicos propios de la evolución y desarrollo (pasar de ser niño a adolescente o llegar a ser adulto), o por situaciones sociales relacionados a ellos (dejar la escuela para estudiar en la secundaria, o elegir una carrera universitaria) han sido provechosos para la comprensión del autoconcepto como causa y consecuencia del comportamiento y el bienestar psicológico (Coelho y Romäo, 2017).

En el contexto educativo, los individuos atribuyen mayor valoración al autoconcepto académico, debido a que este dominio permite al estudiante desarrollar una serie de competencias que le serán útiles durante su formación. En este sentido, el autoconcepto compromete dimensiones: emocionales, sociales y cognitivas que permiten al estudiante tener éxito académico y ajuste escolar (Veiga, et al., 2015; Wang y Fredricks, 2014), por las propiedades motivacionales que presenta este dominio en relación al logro (Byrne, 1984).

En el contexto universitario, las investigaciones resaltan la importancia del autoconcepto especialmente en sus dominios académico y familiar (Pinilla, et al., 2015); por su parte Gargallo, et al., (2009) corroboran la influencia del autoconcepto en el rendimiento académico de los estudiantes. Méndez y Gálvez (2018), sobre este aspecto señalan que, aquellos estudiantes que presentan un mayor autoconcepto académico generalmente muestran mayor satisfacción con sus estudios y capacidad de liderazgo.

Un estudio realizado en Italia por Sagone y De Caroli (2014) con una muestra de 267 estudiantes de diferentes carreras universitarias (Psicología, Medicina y Derecho), demostró que el autoconcepto positivo de los participantes estaba relacionado positivamente con un locus de control interno, mientras que el autoconcepto negativo estaría relacionado positivamente 
con un locus de control externo; las autoras además indican que los niveles de autoeficacia influyen en el autoconcepto de los estudiantes, así, quienes presentaban autoconcepto positivo se percibían a sí mismos como estudiantes autoeficaces, a diferencia de los que presentaban autoconcepto negativo.

$\mathrm{Al}$ ser el autoconcepto un aspecto clave para el bienestar psicológico de una persona, se constituye en una de las principales bases del proceso de formación en los estudiantes universitarios, en razón de que varias de sus competencias se desarrollarán a partir de las actitudes personales que se promuevan en su etapa de preparación profesional (Esnaola, et al., 2008; Pinilla, et al., 2015). A partir de esta premisa, la presente investigación responde al objetivo de evaluar el autoconcepto de los estudiantes de Psicología de la Universidad Técnica de Machala desde una perspectiva multidimensional, debido a que se miden los dominios académico-laboral, social, emocional, familiar y físico.

\section{MATERIALES Y MÉTODOS}

La presente investigación es descriptiva de corte transversal con enfoque cuantitativo (Rubio, 2016; Rodríguez y Mendivelso, 2018). Se utilizó el test AF-5 (Autoconcepto Forma 5) en una muestra de 450 estudiantes, de los cuales 330 fueron mujeres y 120 varones; el promedio de edad estuvo en un rango de 19 a 22 años. En relación a la carrera universitaria a la que pertenecen, 354 corresponden a la carrera de Psicología Clínica y 96 a la carrera de Psicopedagogía, ambas de la Universidad Técnica de Machala. Se consideró además en este estudio dividir la muestra en dos categorías: los de niveles inferiores ( $1^{\circ}$ al $4^{\circ}$ semestre) y los de niveles superiores ( $5^{\circ}$ al $8^{\circ}$ semestre); para los de semestres inferiores la cantidad de estudiantes fue de 293 y para los semestres superiores de 157.

El AF-5 de García y Musito, presentado en sus diversas actualizaciones, en 1999, 2001, 2009 y 2014, es uno de los instrumentos más utilizados en idioma español para medir el autoconcepto desde una perspectiva multidimensional; ha sido validado para ser empleado en países como Ecuador, Colombia, México, Perú, Venezuela, entre otros (García y Musito, 2014). Evalúa en un total de 30 ítems, 5 dimensiones: académico-laboral (ítems: 1, 6, 11, 16, 21, y 26), social (ítems: 2, 7, 12, 17, 22, y 27), emocional (ítems: 3, 8, 13, 18, 23, y 28), familiar (ítems: 4, 9, 14, 19, 24, 29), y físico (ítems: 5, 10, 15, 20, 25, 30). Este instrumento en su versión 2014 permite respuestas en un rango que va desde 1 (en total desacuerdo) hasta 99 (totalmente de acuerdo), y que, para su corrección se debe restar la puntuación obtenida en los ítems 4, 12, 14, y 22 al valor de 100 .

Para efectos de la interpretación de los datos, se han considerado las medias de los percentiles (Pc en adelante) para cada dimensión, tomando en 
cuenta variables como el sexo, la edad de los sujetos estudiados, la carrera a la que pertenecen y el semestre que cursan. Se tomó en cuenta además la propuesta de Parra, et al., (2015), quienes señalan que un estudiante tiene un autoconcepto alto en una dimensión cuando su puntaje es superior a la media del baremo internacional, según lo especificado en el manual de la prueba AF5 para población universitaria. El baremo permite la transformación de los porcentajes directos en porcentajes derivados para ser interpretados estadísticamente. De esta manera, el Pc 50 se constituye en el punto de referencia para la distinción entre un autoconcepto alto o bajo. Adicionalmente, $a$ más del análisis descriptivo, para identificar la existencia de diferencias significativas de las dimensiones del autoconcepto entre las variables, se aplicó para el caso de la variable edad que posee más de dos grupos (19, 20, 21, y 22 años) el método de ANOVA $^{1}$ (análisis de varianzas) (Supriyadi, 2021), mientras que, para las variables sexo (hombre y mujer), carrera (Psicología Clínica y Psicopedagogía) y nivel de estudios (semestres inferiores y semestres superiores), que únicamente tienen dos grupos, se usó la prueba T-Student para muestras/grupos independientes (Navarro, Flores, y González, 2019). Estos métodos gozan de adecuada aceptación y adaptabilidad, debido a que recogen información de experimentos aleatorios (Melo, et al., 2007). El análisis estadístico se realizó utilizando el programa SPSS versión 24.

\section{RESULTADOS Y DISCUSIÓN}

Una vez aplicado el instrumento a la muestra objeto de estudio y hecho el análisis estadístico pertinente, se presentan los siguientes resultados:

Analizando los datos de los puntajes obtenidos por los estudiantes en las cinco dimensiones evaluadas (Tabla 1), se puede manifestar que en cuatro de ellas no existieron diferencias significativas asociadas a la edad, sexo, carrera o nivel de estudio. Únicamente en la dimensión familiar se revelaron diferencias estadísticamente significativas cuando se comparó las puntuaciones de la variable sexo ( $p$-valor $=0,042$ ) y carrera ( $p$-valor $=0,049$ ), siendo que, para las categorías de sexo, los hombres presentaron niveles de autoconcepto familiar más altos $(\mathrm{Pc}=78)$ que las mujeres $\left(\mathrm{Pc}_{\mathrm{C}}=47\right)$. Del mismo modo, en cuanto al tipo de carrera a la que pertenecen los estudiantes, los mejores puntajes se presentaron en aquellos que pertenecen a la carrera de Psicología Clínica $(\mathrm{Pc}=77)$, versus a aquellos que cursan psicopedagogía $(\mathrm{Pc}=46)$.

${ }^{1}$ Se aplicó a posteriori de verificar el cumplimiento de normalidad (Kolmogorov-Smirnov=0,084), homocedasticidad (Levene $=0,057$ ), e independencia de factores (Barrios y Silva, 2019).

\section{Cumbres}


Tabla 1

Diferencia de medias de las dimensiones del AF-5 Autoconcepto

\begin{tabular}{|c|c|c|c|c|c|c|c|c|}
\hline \multirow{2}{*}{ DIMENSIONES } & \multicolumn{2}{|c|}{ Edad } & \multicolumn{2}{|c|}{ Sexo } & \multicolumn{2}{|c|}{ Carrera } & \multicolumn{2}{|c|}{$\begin{array}{l}\text { Nivel de } \\
\text { estudio }\end{array}$} \\
\hline & ANOVA & $\begin{array}{l}\text { Media } \\
(\mathrm{Pc})\end{array}$ & $\begin{array}{l}\text { T de } \\
\text { Student }\end{array}$ & $\begin{array}{l}\text { Media } \\
(\mathrm{Pc})\end{array}$ & $\begin{array}{l}\mathrm{T} \text { de } \\
\text { Student }\end{array}$ & $\begin{array}{l}\text { Media } \\
(\mathrm{Pc})\end{array}$ & $\begin{array}{l}\text { T de } \\
\text { Student }\end{array}$ & $\begin{array}{l}\text { Media } \\
(\mathrm{Pc})\end{array}$ \\
\hline $\begin{array}{l}\text { Académico/ } \\
\text { laboral }\end{array}$ & 0,123 & $\begin{array}{l}49 \\
55 \\
60 \\
48\end{array}$ & 0,401 & $\begin{array}{l}78 \\
49\end{array}$ & 0,509 & $\begin{array}{l}60 \\
48\end{array}$ & 0,326 & $\begin{array}{l}66 \\
52\end{array}$ \\
\hline Social & 0,224 & $\begin{array}{l}65 \\
64 \\
64 \\
68\end{array}$ & 0,209 & $\begin{array}{l}68 \\
49\end{array}$ & 0,653 & $\begin{array}{l}71 \\
49\end{array}$ & 0,311 & $\begin{array}{l}73 \\
46\end{array}$ \\
\hline Emocional & 0,213 & $\begin{array}{l}62 \\
70 \\
65 \\
55\end{array}$ & 0,376 & $\begin{array}{l}73 \\
79\end{array}$ & 0,329 & $\begin{array}{l}66 \\
54\end{array}$ & 0,091 & $\begin{array}{l}75 \\
60\end{array}$ \\
\hline Familiar & 0,431 & $\begin{array}{l}71 \\
68 \\
55 \\
64\end{array}$ & 0,042 & $\begin{array}{l}78 \\
47\end{array}$ & 0,049 & $\begin{array}{r}77 \\
46\end{array}$ & 0,451 & $\begin{array}{l}65 \\
40\end{array}$ \\
\hline Físico & 0,287 & $\begin{array}{l}65 \\
45 \\
55 \\
69\end{array}$ & 0,087 & $\begin{array}{l}72 \\
56\end{array}$ & 0,148 & $\begin{array}{l}74 \\
48\end{array}$ & 0,671 & $\begin{array}{l}71 \\
49\end{array}$ \\
\hline
\end{tabular}

Nota: La información mostrada respectivamente y de forma vertical, la variable edad: 19, 20, 21, y 22 años; variable sexo: Hombres, y mujeres; variable carrera: Psicología Clínica, y Psicopedagogía; y variable nivel de estudios: superiores, e inferiores.

En relación a la edad, pese a que se encontró que no existe diferencia estadísticamente significativa entre sus categorías, en términos de media, se reveló que los universitarios que poseen una edad de 19 años tienen mejores valores $(\mathrm{Pc}=71)$ en la dimensión familiar, mientras que los que tienen 20 $(\mathrm{Pc}=70)$ y 21 años $(\mathrm{Pc}=65)$ predominan con un puntaje más alto en la parte emocional; en cambio, los alumnos que tienen una edad de 22 años $(\mathrm{Pc}=69)$ puntuaron más alto dentro de la dimensión físico.

$\mathrm{Al}$ respecto, diversas investigaciones indican que el autoconcepto presenta variaciones en relación a la edad de la persona, Marsh (1989) sugiere que existe una declinación en la adolescencia temprana, mientras que en la adolescencia media y adultez temprana se estabiliza. Por su parte, Shavelson, et al., (1976) manifiestan que a mayor edad, las personas son capaces de diferenciar sus autoevaluaciones entre los diferentes dominios del autoconcepto, así como también Marsh y Ayote (2003), proponen la hipótesis diferencial distintiva en la que mencionan que la edad y desarrollo cognitivo en el individuo permite que el autoconcepto se integre desde sus elementos similares y se diferencie con mayor claridad en sus aspectos disímiles. 
Por otra parte, atendiendo a la categorización de los niveles de estudio como "superiores o inferiores" se encontró que, los mejores valores estaban asentados en la dimensión emocional, y dentro de esta, fueron los estudiantes de los niveles superiores quienes evidenciaron mejor puntuación $\left(\mathrm{Pc}_{\mathrm{C}}=75\right)$, que aquellos de niveles inferiores ( $\mathrm{Pc}=60)$. En consonancia, Pinilla, et al., (2015) mencionan que el autoconcepto emocional como percepción negativa respecto al control de sus propias emociones puede estar asociado a las dificultades que experimentan los estudiantes al iniciar su vida académica y adaptarse a las exigencias del contexto universitario. Autores como García y Musitu (2009) y Véliz (2010) exponen que el autoconcepto suele ser más estable y fuerte en la medida en que se avanza en el ciclo vital y en el curso de una carrera universitaria.

Así mismo, Burgos y Apocada (2012) en un estudio cuyo objetivo fue determinar los niveles de autoconcepto, autoeficacia académica y bienestar psicológico en una muestra de estudiantes universitarios chilenos, concluyeron que en la medida que se tiene mayor edad en la universidad se tiende a tener una mayor percepción positiva de las capacidades individuales para el éxito universitario.

Teniendo en cuenta que el instrumento utilizado en la presente investigación se sustenta en un modelo multidimensional de autoconcepto, el de Shavelson, et al., (1976), y reiterando lo expuesto en la metodología que para efectos de la interpretación de los datos, se ha considerado que un estudiante tiene un autoconcepto alto en una dimensión, cuando su puntaje en ella es superior a la media del baremo internacional de la prueba AF5 para población universitaria. A continuación, se presentan datos sobre cada una de las dimensiones del autoconcepto evaluadas, para su posterior interpretación:

Tabla 2

Niveles del autoconcepto por dimensiones según percentiles

\begin{tabular}{|l|l|}
\hline DIMENSIONES & \multicolumn{1}{l|}{ NIVELES } \\
\hline Académico-laboral & $86,6 \%$ - Alto \\
& $13,4 \%$ - Bajo \\
\hline Social & $56,6 \%$ - Alto \\
& $43,4 \%$ - Bajo \\
\hline Emocional & $54,5 \%$ - Alto \\
& $45,5 \%$ - Bajo \\
\hline Familiar & $2 \%$ - Alto \\
& $98 \%$ - Bajo \\
\hline Físico & $78,7 \%$ - Alto \\
& $21,3 \%$ - Bajo \\
\hline
\end{tabular}

Nota: Se considera al Pc 50 como referente para clasificar en alto (Pc $>50)$ o bajo (Pc $\leq 50)$ el autoconcepto de los universitarios. 
Haciendo un análisis sobre los niveles de autoconcepto de los sujetos estudiados, se manifiesta que, en cuatro de las cinco dimensiones evaluadas los porcentajes fueron superiores a la media, siendo los de mayor puntuación las dimensiones académico laboral y la correspondiente al autoconcepto físico; situación que no se muestra disímil a lo expresado por autores como Gargallo, et al., (2009) y Pinilla, et. al (2015), quienes afirman que una de las dimensiones del autoconcepto en la que los estudiantes universitarios tienen una imagen más positiva de sí mismos es la dimensión académico laboral. Con respecto al autoconcepto físico, el hecho de que los sujetos investigados sean adultos jóvenes universitarios explica que las puntuaciones sean altas ya que los sujetos jóvenes tienden a percibirse más positivamente en algunos aspectos de esta dimensión, especialmente en lo que se refiere a su condición física, atractivo y salud (García y Musitu, 2014).

Las puntuaciones en las dimensiones que corresponden al autoconcepto social y emocional no presentan una diferencia en términos medios como para ser consideradas representativas de un autoconcepto alto en la población total estudiada. Finalmente se puede evidenciar diferencia en las puntuaciones que marcan la existencia de un autoconcepto considerado como alto o bajo en la dimensión familiar, resultando que los estudiantes presentan un autoconcepto bajo en esta dimensión. Hallazgo que resulta similar al llevado a cabo por Parra, et al., (2015) en el que se evaluó el autoconcepto de los estudiantes de la universidad de Antioquia-Colombia, a través de la prueba AF5, en donde la dimensión familiar se ubicó por debajo del Pc 50 en 11 de 13 carreras estudiadas, siendo ésta la dimensión con los puntajes más bajos.

Siendo el autoconcepto familiar una de las dimensiones fundamentales debido a que correlaciona positivamente con las demás dimensiones (García y Musitu, 2009), es indispensable que futuras investigaciones procuren afianzar hallazgos en cuanto a factores causales, análisis diferenciales y programas interventivos, que evalúen e incidan sobre las percepciones que tienen los estudiantes respecto a su implicación, participación e integración en el medio familiar, sobre todo de la confianza y el apoyo percibido en relación a su desenvolvimiento en la vida académica y futuro profesional.

\section{CONCLUSIONES}

Los estudiantes de Psicología de la Universidad Técnica de Machala presentaron un porcentaje mayor de puntuaciones superiores a la media en cuatro de las cinco dimensiones del autoconcepto evaluadas; los niveles más altos de autoconcepto se evidenciaron en las dimensiones correspondientes a lo académico-laboral y físico. Las puntuaciones en las dimensiones social y emocional no fueron estadísticamente representativas de un autoconcepto alto para la población total estudiada.

Se revelaron puntuaciones considerablemente bajas en la dimensión correspondiente al autoconcepto familiar, requiriéndose nuevas investigaciones en esta área para afianzar los hallazgos en términos de análisis diferenciales, factores causales y programas interventivos. Únicamente en esta dimensión 
del autoconcepto, se revelaron diferencias significativas relacionadas al sexo y a la carrera, siendo los estudiantes de la carrera de Psicopedagogía y las mujeres quienes presentan niveles más bajos.

Por otra parte, los estudiantes de los semestres inferiores presentaron niveles bajos de autoconcepto en relación a los semestres superiores en lo concerniente a la dimensión emocional. Finalmente, en relación a la edad no hubo diferencias significativas en las distintas dimensiones del autoconcepto.

En cuanto a las limitaciones de este estudio, se puede mencionar que al ser el autoconcepto una variable psicológica subjetiva, los resultados podrían ser más profundos si se le evalúa desde un enfoque de investigación cualitativa; e incluso empleando un diseño longitudinal de panel, se determinaría con mayor certeza si el autoconcepto de los estudiantes universitarios varía o se modifica conforme avanzan en sus estudios profesionales. Además, el haber evaluado el autoconcepto únicamente en dos carreras y de una misma institución, limita la riqueza de la investigación en términos de comparación.

\section{REFERENCIAS BIBLIOGRÁFICAS}

Barrios, R., \& Silva, R. (2019). Pertinencia de uso del análisis estadístico de medidas repetidas en la investigación agrícola. Agronomía Tropical, 69(1), 9-17.

Byrne, B. (1984). The general/academic self-concept nomological network: a review of construct validation research. Review of Educational Research, (54), 427-456.

Campbell, J., Trapnell, P., Heine, S., Katz, I., Lavallee, L. y Lehman, D. (1996). Self-concept clarity: Measurement, personality correlates, and cultural boundaries. Journal of Personality and Social Psychology, 70(1), 141-156.

Cazalla, L. y Molero, D. (2013). Revisión teórica sobre el autoconcepto y su importancia en la adolescencia. Revista Electrónica de Investigación y Docencia, (10), 43-64.

Chaparro-Aguado, F. (2020). The game board in the university classroom: perception of the students. Education, Sport, Health and Physical Activity, 4(1), 63-82. https://core.ac.uk > download > pdf

Coelho, V. y Romäo, A. (2017). The impact of secondary school transition on self-concept and self-steem. Revista de Psicodidáctica, 22(2), 85-92.

Cole, D., Maxwell, S., Martin, J., Peeke, L., Seroczynski, A., Tram, J. y Maschman, T. (2001). The development of multiple domains of child and adolescent self-concept: a cohort sequential longitudinal design. Child development, 72, 1723-1746.

Esnaola, I., Goñi, A. y Madariaga, J. (2008). El autoconcepto: Perspetivas de investigación. Revista de Psicodidáctica, 13(1), 179-194.

Garaigordobil, M. y Durá, A. (2005). Neosexismo en adolescentes de 14 a 17 años: relaciones con autoconcepto-autoestima, personalidad, psicopatología, problemas de conducta y habilidades sociales. Clinica y Salud, 17(2), 127-149.

\section{Cumbres}


García, F. y Musitu, G. (1999). AF5: Autoconcepto Forma 5 . Madrid: TEA.

García, F. y Musitu, G. (2014). AF5: Autoconcepto forma 5. Madrid, España: TEA.

Gargallo López, B., Garfella Esteban, P. R., Sánchez Peris, F., Ros Ros, C. y Serra Carbonell, B. (2009). La influencia del autoconcepto académico en el rendimiento académico de estudiantes universitarios. Revista Española de Orientación y Psicopedagía, 20(1), 16-28.

González, F. (2005). Estructura multidimensional del autoconcepto físico. Revista de Psicodidáctica, 10(1), 121-130.

Jansen, M., Scherer, R., y Schroeders, U. (2015). Students' self-concept and self-efficacy in the sciences: Differential relations to antecedents and educational outcomes. Contemporary Educational Psychology, 41, 13-24.

López Manriquea, I., González González de Mesab, C., San Pedro Veledoa, B. y San Pedro Veledoa, J. (2016). Del autoconcepto general al autoconcepto artístico. Magister, 18(1), 42-49. doi:http://dx.doi.org/10.1016/j.magis.2016.06.002

Marsh, H., y Shavelson, R. (1985). Self-concept: it's multi-faceted, hierarchical structure. Educational Psychologist, 20, 107-123. doi:https://doi. org/10.1207/s15326985ep2003_1

Marsh, H. (1989). Age and sex effects in multiple dimensions of self-concept: preadolescence to early adulthood. Journal of Educational Psychology, 81, 417-430.

Marsh, H., y Ayotte, V. (2003). Do Multiple Dimensions of Self-Concept Become More Differentiated With Age? The Differential Distinctiveness Hypothesis. Journal of Educational Psychology, 95(4), 687-706. doi:http://dx. doi.org/10.1037/0022-0663.95.4.687

Mato Medina, O., Ambris Sandoval, J., Llergo Young, M. y Mato Martínez, Y. (2020). Autoconcepto en adolescentes considerando el género y el rendimiento académico en Educación Física. Universidad y Sociedad,, 12(6), 22-30. https://rus.ucf.edu.cu/index.php/rus/article/view/1806/1802

Melo, O., López, L. y Melo, S. (2007). Diseño de bloques completamente aleatorizados. En O. Melo, L. López, y S. Melo, Diseño de experimentos [Métodos y aplicaciones] (p. 161). Bogotá: Pro-Offset Editorial S.A.

Méndez Vera, J., y Gálvez Nieto, J. (2018). Propiedades psicométricas de la Escala de Autoconcepto Académico (EAA) en estudiantes universitarios chilenos. Liberabit, 24(1), 131-145. doi:10.24265/liberabit.2018.v24n1.09

Navarro, G., Flores, G., \& González, M. (2019). Diferencias por sexo en el nivel de Autoeficacia percibida en una muestra de estudiantes de la Provincia de Concepción ¿Qué papel juegan los roles de género en la educación? Educación inclusiva, 12(1), 205-224. Obtenido de https://revistaeducacioninclusiva.es/index.php/REI/article/view/389/374

Obregón-Brocher, N., Armenta-Hurtarte, C., Arari-Zayat, D. y Ortiz-Izquierdo Herington, R. (2020). Maternidad cuestionada. Revista de Psicología, 19(1), 104-119. doi:10.24215/2422572Xe047 
Palacios-Garay, J., y Coveñas-Lalupú, J. (2019). Predominancia del autoconcepto en estudiantes con conductas antisociales del Callao. Revista Propósitos y Representaciones, 7(2), 325-352. doi:http://dx.doi.org/10.20511/ pyr2019.v7n2.278

Parra, C., Restrepo, G., Usuga, O., Castañeda, E., Estrada, P., M., Uñates, E., Gil, A. y Mendoza, R. (2015). Relación del Autoconcepto y del acompañamiento psicopedagógico con el rendimiento académico en estudiantes de primer semestre de ingeniería. Revista Ingeniería y Sociedad, 9, 40-66.

Penado, M. y Rodicio, M. (2017). Análisis del autoconcepto en las víctimas de violencia de género entre adolescentes. Suma Psicológica, 24(2), 107-114. https://www.sciencedirect.com/science/article/pii/S012143811730022X\#bib0020

Pekrun, R., y Stephens, E. (2015). Self-Concepts: Educational Aspects. doi:https://doi.org/10.1016/B978-0-08-097086-8.92018-X

Pilarska, A. (2018). Big-five personality and aspects of the self-concept: variable- and person centered approaches. Personality and Individual Differences, 127, 107-113.

Pinilla, V., Montoya, D. y Dussán, C. (2015). Caracterización del Autoconcepto en una muestra de estudiantes universitarias del Programa de Desarrollo Familiar de Manizales, Colombia. Revista Psicogente.

Rodríguez, M. y Mendivelso, F. (2018). Diseño de investigación de corte transversal. Rev.Medica.Sanitas(21), 141-146. https://www.unisanitas.edu.co/ Revista/68/07Rev\%20Medica\%20Sanitas\%2021-3_MRodriguez_et_al.pdf

Rubio, S. (2016). Sección de metodología. Enfermería basada en la evidencia: Investigación clínica aplicada a las ciencias de la salud. Enfermería en Cardiología, 13(69), 23-29. https://dialnet.unirioja.es/descarga/articulo/6285990.pdf

Sagone, E. y De Caroli, M. (2014). Locus of control and academic self-efficacy in university students: the effects of Self-concepts. Procedia - Social and Behavioral Sciences, 114, 222 - 228.

Shavelson, R., Hubner, J., y Stanton, G. (1976). Self-concept: validation of construct interpretations. Review of Educational Research, 46, 407-441.

Supriyadi, E. (2021). Analisis pengendalian kualitas produk dengan statistical proses control (SPC) di PT. Surva Toto Indonesia, Tbk. JITMI, 1(1), 63-73. Obtenido de http://www.openjournal.unpam.ac.id/index.php/JITM/ article/view/1410/1105

Veiga, F., García, F., Reeve, J., Wentzel, Kathryn, y García, Ó. (2015). Cuando se pierde la motivación escolar de los adolescentes con mejor autoconcepto. Revista de Psicodidáctica, 20(2), 305-320.

Véliz, B. (2010). Dimensiones del autoconcepto en estudiantes chilenos: Un estudio psicométrico. Bilbao: Universidad del País Vasco.

Wang, M. y Fredricks, J. (2014). The reciprocal links between school engagement, youth problem behaviors, and school dropout during adolescence. Child Development, 85(2), 722-737. doi:10.1111/cdev.12138. Epub 2013 Jul 29.

\section{Cumbres}

